\title{
Yoga and acupuncture versus "sham" treatments for menopausal hot flashes: how do they compare?
}

Amanda McGuire, PhD, and Debra Anderson, PhD

Women experiencing transition to menopause frequently report troubling vasomotor symptoms (VMS), especially hot flashes.1 Although hormone therapy $(\mathrm{HT})$ is an effective pharmacological treatment, many women prefer nonpharmacological options. ${ }^{2}$ Furthermore, HT is contraindicated in some women and not without risk. ${ }^{3}$ For example, in women with existing cardiovascular disease or recent breast cancer, HT may not be an option. ${ }^{4}$

There is evidence that acupuncture is effective in reducing the frequency and duration of hot flashes. ${ }^{5}$ Similarly, yoga appears to be a safe and effective means of reducing menopausal symptoms comparable to other exercise interventions, but with an added effect on VMS. ${ }^{6}$ The article published in this issue of Menopause by Avis et al ${ }^{7}$ confirms the benefits of acupuncture and yoga on hot flashes, but also reports similar positive effects in attention control groups receiving either sham acupuncture or health and wellness education, compared to usual care and waitlist control.

The article reports results of a pooled analysis of three trials with a total enrolment of 319 perimenopausal and postmenopausal women experiencing four or more hot flashes per day and not currently taking HT, with the primary outcome measure being percentage reduction in self- reported daily hot flashes. When comparing three active interventions (one yoga, two acupuncture), two attention control groups (sham acupuncture, health and wellness education classes), and three passive control groups (one usual care, two waitlist) they found similar significant effects on hot flashes in all active intervention and attention control groups over 8 weeks. Interestingly, the attention control groups were equally effective to the actual yoga and acupuncture treatments.

The authors pose a number of possible explanations including the attention control conditions being active treatments; the effects of patient provider interactions; and treatment expectations/placebo effect. The sham acupuncture involved shallow needling twice a week for 8 weeks (30- minute treatment

sessions), whereas the health and wellness education included 10 weekly, 90-minute didactic teaching about a range of health topics. With participants in both of these groups having high interaction with treatment conditions and health providers over 8 to 10 weeks the author's explanations are plausible.

In regard to the health and wellness education sessions in particular, while they did not specifically address manage- ment of hot flashes, a wide range of health topics was covered including menopause, stress management, bone health, cardiovascular health, nutrition, and aging. It seems likely that motivated participants might also undertake further self-directed reading about management of hot flashes and potentially made behavioral changes as a result. Furthermore, with evidence that individual attitude to menopause can affect symptom experience, ${ }^{8}$ the social support women receive from other group members over the 10 weeks, might also contribute to normalizing the experience for women in this age group.

In conclusion, Avis and colleagues highlight how attention control groups can complicate interpretation of 
results and the necessity to include passive control groups in behavioral intervention trials. This study also confirms the benefits of yoga and acupuncture for management of hot flashes. Importantly, results in this study suggest that tailored health and wellness education classes for midlife women also hold promise as an effective nonpharmacological intervention for management of VMS.

\section{REFERENCES}

1. Blumel JE, Chedraui P, Baron G, et al. A large multinational study of vasomotor symptom prevalence, duration, and impact on quality of life in middle-aged women. Menopause 2011;18:778-785.

2. Daley A, Stokes-Lampard H, Macarthur C. Exercise for vasomotor meno- pausal symptoms. Cochrane Database Syst Rev 2011;5:CD006108.

3. Hamoda H, Savvas M, Panay N. New guidelines for hormone-replacement therapy: an update on risks and benefits for clinical practice. Clin Pract 2013;10:675-678.

4. MacLennan AH. HRT in difficult circumstances: are there any absolute contraindications? Climacteric 2011;14:409-417.

5. Chiu H-Y, Pan C-H, Shyu Y-K, Han B-C, Tsai P-S. Effects of acupuncture on menopause-related symptoms and quality of life in women in natural menopause: a meta-analysis of randomized controlled trials. Menopause 2015;22:234-244.

6. Cramer H, Peng W, Lauche R. Yoga for menopausal symptoms-a systematic review and meta-analysis. Maturitas 2018;109:13-25.

7. Avis NE, Levine BJ, Danhauer S, Coeytaux RR. A pooled analysis of three studies of nonpharmacological interventions for menopausal hot flashes. Menopause 2019;26:350-356.

8. Ayers B, Forshaw M, Hunter MS. The impact of attitudes towards the menopause on women's symptom experience: a systematic review. Maturitas 2009;65:28-36. 